frequency fast spikes and polyspikes interspersed with fast low amplitude activity, and MRI signal alterations with thickening of cortex, blurred grey-white matter junction and hyperintensity of subcortical white matter. Group 3 Taylortype had the best outcome, with $75 \%$ seizure-free after one year follow-up, compared with $50 \%$ in type 2 cytoarchitectural cases, and $43 \%$ seizure-free in type 1 architectural dysplasia cases. The three-category classification has easily recognized histopathological findings, and clinically homogeneous characteristics. (Tassi L, Colombo N, Garbelli R et al. Focal cortical dysplasia: n europathological subtypes, EEG, neuroimaging and surgical outcome. Brain Auguist 2002;125:1719-1732). (Respond: Robert Spreafico MD PhD, Department of Experimental Neurophysiology and Epileptology, Instituto Nazionale Neurologico 'C. Besta', Via Celoria 11, 20133 Milano, Italy).

COMMENT. In the above series of focal cortical dysplasia, the video or stereo-EEG provided the best delineation of the epileptogenic zone, superior to the MRI. In one-third of cases, the MRI was unremarkable, and in those with MRI evidence of a lesion, the boundaries were ill-defined. Type 3 Taylor-type dysplasia

had a better outcome than the type 1 architectural and type 2 cytoarchitectural dysplasias.

\title{
HEREDITARY SUSCEPTIBILITY TO FETAL VALPROATE SYNDROME
}

Three families in whom all 7 siblings were affected by fetal valproate syndrome (FVS) are reported from the Department of Medical Genetics, Family Federation of Finland, Helsinki, Finland. The parents of affected children had normal intelligence and were nonconsanguinous. Maternal epilepsy was idiopathic, myoclonus or secondary to arteriovenous malformation. Maternal age ranged from 23 to 34 . Daily valproate dosage ranged from a low of $1000-1200 \mathrm{mg}$ to $2000-2500 \mathrm{mg}$, with serum valproate levels of $41-46$ to $77-101 \mathrm{mg} / \mathrm{L}$. Age at examination of the 7 affected children varied from 1 yr 5 months to 8 years 0 months. Developmental deficit was mild to moderate in all patients. Malformations involved the face, urinary tract, cleft palate, hands (small finger nails) and feet, and inguinal hernias or undescended testis in all. Craniosynostosis (trigonocephaly) occurred in 2, tracheomalacia and ventriculomegaly in 1 , and severe hearing deficit in 1 . No neural tube defects occurred in these patients, only 2 of the mothers having received folic acid, only 1 prior to conception. Neonatal VPA withdrawal symptoms (convulsions, feeding problems or respiratory distess) occurred in 5 infants; these resolved promptly with treatment and did not account for the developmental delay. The occurrence of FVS in all the siblings strongly suggests an hereditary susceptibility to valproate embryopathy. (Malm H, Kajantie E, Kivirikko S et al. Valproate embryopathy in three sets of siblings: Further proof of hereditary susceptibility. Neurology August (2 of 2) 2002;59:630-633). (Reprints: Dr Heli Malm, Teratology Information, Department of Medical Genetics, Family Federation of Finland, PO Box 849, Helsinki, FIN00101, Finland).

COMMENT. Hereditary susceptibility to fetal valproate syndrome (FVS) is suggested by this report of three families with all siblings affected. FVS is characterized by typical facial features (medial deficiency of eyebrows, broad nasal root, anteverted nares, shallow philtrum, and long and thin upper lip), trigonocephaly, developmental delay, neural tube, cardiac and genitourinary malformations, and limb defects. The risk of recurrence in a subsequent pregnancy may be high, indicating avoidance of further VPA therapy. 